SCIENTIFIC LETTER

\title{
Time course of transcardiac interleukin- 6 release after coronary stenting for stable angina
}

\author{
J M Kefer, L M Galanti, C E Hanet
}

Heart 2004;90:943-944. doi: 10.1136/hrt.2003.016105

C ytokines are known to play a critical role in the atherosclerotic process. ${ }^{1}$ The cytokine interleukin-6 (IL-6) is involved in a variety of physiological functions ultimately leading to macrophage activation, platelet aggregation, and stimulation of matrix degrading enzymes. An increase in circulating concentrations of IL-6 and of its hepatic byproduct $\mathrm{C}$ reactive protein (CRP) has been reported in unstable angina ${ }^{2}$ and after percutaneous coronary intervention (PCI); high concentrations of IL-6 and CRP are considered to be markers of poor prognosis, probably related to the intensity of plaque inflammation. The present study was designed specifically to evaluate whether transmyocardial production of IL-6 could be measured immediately and within two hours of a successful elective PCI and stent implantation in stable patients.

\section{METHODS}

The study involved 11 patients with stable angina, admitted for an elective PCI of the proximal segment of the left anterior descending coronary artery. All patients received standard medical treatment consisting of intravenous heparin (70 U/kg body weight), acetylsalicylic acid, and nitrates. No glycoprotein IIb/IIIa inhibitors were given. A six French Cournand catheter was introduced through the right femoral vein in the coronary sinus and the left coronary ostium was catheterised through the right femoral artery. Coronary sinus and arterial blood samples were taken simultaneously at baseline, immediately after stent deployment and two hours later to determine IL-6 and lactate concentration. Additional blood samples were obtained from a peripheral vein before the procedure for measurement of CRP and troponin I circulating concentrations and also 12 hours later for measurement of troponin-I concentration.

\section{RESULTS}

At baseline, mean (SD) IL-6 concentrations were similar in the aorta and in the coronary sinus $(3.82$ (3.25) $v 3.84$ (3.09) $\mathrm{pg} / \mathrm{ml}$ respectively, not significant), the coronary sinus to aorta ratio averaging $1.02(0.13)$. A transcardiac extraction of lactate was observed in all cases, with a coronary sinus to aorta ratio averaging $0.77(0.22)$ and mean serum concentrations of $0.61(0.16) \mathrm{mM} / \mathrm{l}$ in the aorta and 0.47 $(0.21) \mathrm{mM} / \mathrm{l}$ in the coronary sinus. The systemic troponin I concentration was within the normal range in all patients (0.03 $(0.01) \mathrm{ng} / \mathrm{ml})$ and CRP concentration averaged 0.28 (0.20) $\mathrm{mg} / \mathrm{dl}$, confirming the stability of the coronary disease. Immediately after stenting, blood sampled in the coronary sinus during the hyperaemic phase following balloon deflation showed a consistent increase in lactate concentration (1.38 (0.76) mM/l, p $=0.0008 v$ baseline), the lactate coronary sinus to aorta ratio increasing significantly to 2.42 (1.06) $(p=0.0001)$. Simultaneously, the IL-6 coronary sinus to aorta ratio (fig 1) increased in all but one patient (mean 1.17 (0.17), not significant $v$ baseline) but these changes remained insignificant. Two hours after the procedure, IL- 6 concentrations increased both in the aorta and in

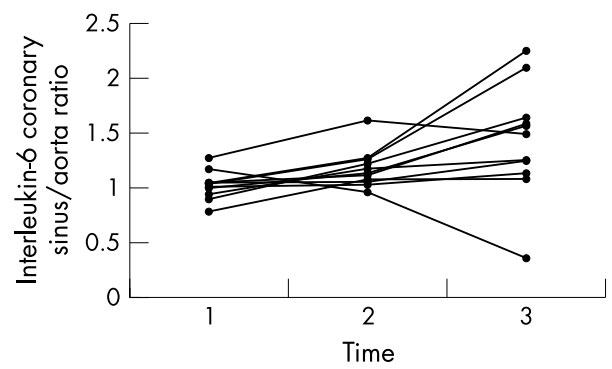

Figure 1 Individual changes in interleukin-6 coronary sinus to aorta ratio. Time 1 = baseline; time 2 = immediately after stenting; time $3=$ 2 hours after stenting.

the coronary sinus (7.33 (5.20) and 8.67 (4.89) pg/ml, $\mathrm{p}<0.01 v$ baseline), the coronary sinus to aorta ratio demonstrating a sustained transcardiac release (1.42 (0.50), $\mathrm{p}=0.02 v$ baseline). No increase in troponin I was observed 12 hours after the procedure $(0.05 \quad(0.01) \mathrm{ng} / \mathrm{ml}$; not significant $v$ baseline).

\section{DISCUSSION}

This study demonstrates that the increase in circulating IL-6 concentration observed after coronary angioplasty results from a transmyocardial production occurring within the territory of the treated coronary vessel and is independent of any detectable sign of necrosis. This IL-6 release is significant two hours after the procedure, with a modest and insignificant increase being observed in the first seconds immediately following stent implantation.

Inflammation is an initial consequence of an angioplasty procedure, possibly triggered by the exposure of the thrombogenic surface of the vessel wall to circulating leucocytes and by the recruitment of inflammatory cells from the overstretched adventitia. In addition, stent deployment can cause a foreign body reaction, amplifying the inflammatory response.

Previous studies designed to measure the expression of inflammatory markers after angioplasty provided conflicting results. Liuzzo and colleagues ${ }^{3}$ showed that the rise of cytokine concentrations after PCI is determined by a preprocedural individual hyper-responsive immune system and not by the plaque rupture. Cusack and colleagues ${ }^{4}$ showed that the intracardiac production of IL-6 in unstable angina could be the result of low grade myocardial necrosis. In these studies (except for one subgroup of the Cusack study), the blood samples for IL-6 concentration assessment were drawn from a peripheral site and not from the coronary sinus. This method did not allow a precise measurement of local myocardial cytokine production relating to the vascular trauma and the coronary stenting.

Abbreviations: CRP, C reactive protein; IL-6, interleukin-6; $\mathrm{PCI}$, percutaneous coronary intervention 
Hojo and colleagues ${ }^{5}$ demonstrated a relation between the rise of IL-6 concentration in the coronary sinus after PCI and the risk of late restenosis. No late blood sampling and no simultaneous measurement of arterial concentrations of IL-6 were carried out.

In our study, we used a carefully selected population, only including stable patients with a single short stenosis of the proximal segment of the left anterior descending coronary artery. All patients were treated with a single stent implantation in order to produce a standardised inflammatory stimulus. Samples were taken simultaneously from the aorta and the coronary sinus, which is the only method that assesses local transmyocardial production. Moreover, to confirm that the effluent of the treated vascular territory was adequately sampled in the coronary sinus, we measured lactate concentration on arterial and venous blood samples obtained immediately after stent implantation. In these conditions, a modest and insignificant transmyocardial IL-6 release is detectable immediately after stenting, but a sustained transmyocardial production is observed two hours after the procedure which is independent of any systemic troponin I concentration increase. This time course suggests that the increase in IL-6 circulating concentrations after PCI is related to an inflammatory response within the coronary vasculature occurring in the vascular territory directly affected by the procedure, most likely at the site of the stent implantation. The absence of any increase in troponin I concentration shows that this inflammatory reaction is not triggered by necrosis in underlying myocardial areas. The measurement of this local production of inflammatory mediators could help to quantify the reaction to various types of stent materials and the ability of drug eluting stents to prevent local inflammatory reaction after PCI.

\section{Authors' affiliations}

J M Kefer, L M Galanti, C E Hanet, Division of Cardiology, University of Louvain, Brussels, Belgium

Correspondence to: Dr Joëlle Kefer, St-Luc University Hospital, Division of Cardiology, Avenue Hippocrate 10/2881, B-1200 Brussels, Belgium; joelle.kefer@clin.ucl.ac.be

Accepted 6 November 2003

\section{REFERENCES}

1 Hansson GK, Libby P, Schônbeck U, et al. Innate and adaptive immunity in the pathogenesis of atherosclerosis. Circ Res 2002;91:281-91.

2 Ikeda U, Ito T, Shimada K. Interleukin-6 and acute coronary syndrome. Clin Cardiol $2001 ; 24(11): 701-4$.

3 Liuzzo G, Buffon A, Biasucci LM, et al. Enhanced inflammatory response to coronary angioplasty in patients with severe unstable angina. Circulation 1998;22:2370-80

4 Cusack MR, Marber MS, Lambiase PD, et al. Systemic inflammation in unstable angina is the result of myocardial necrosis. J Am Coll Cardiol 2002;39:1917-23.

5 Hojo Y, Ikeda U, Katsuki T, et al. Interleukin 6 expression in coronary circulation after coronary angioplasty as a risk factor for restenosis. Heart 2000;84:83-7.

\section{IMAGES IN CARDIOLOGY}

\section{Aneurysms of coronary artery with thrombus}

A

21 year old man, with no conventional risk factors for coronary artery disease, presented with an acute inferior wall myocardial infarction. The patient was thrombolysed with streptokinase. Transthoracic echocardiography showed good left ventricular function with mild inferior wall hypokinesia. He had no history of Kawasaki's disease. Selective coronary angiogram of the left coronary artery showed diffuse dilatation of the left main and proximal left anterior descending artery, and the proximal circumflex artery (panels A and B). Selective coronary angiogram of the right coronary artery revealed a dilated proximal coronary artery with thrombus (panel C). The patient was treated with aspirin, clopidogrel and warfarin, and the international normalised ratio (INR) maintained at 2.5. A repeat angiogram 40 days later revealed a recanalised right coronary artery (panel D). The patient was discharged on the same drugs and is doing well at six months follow up.
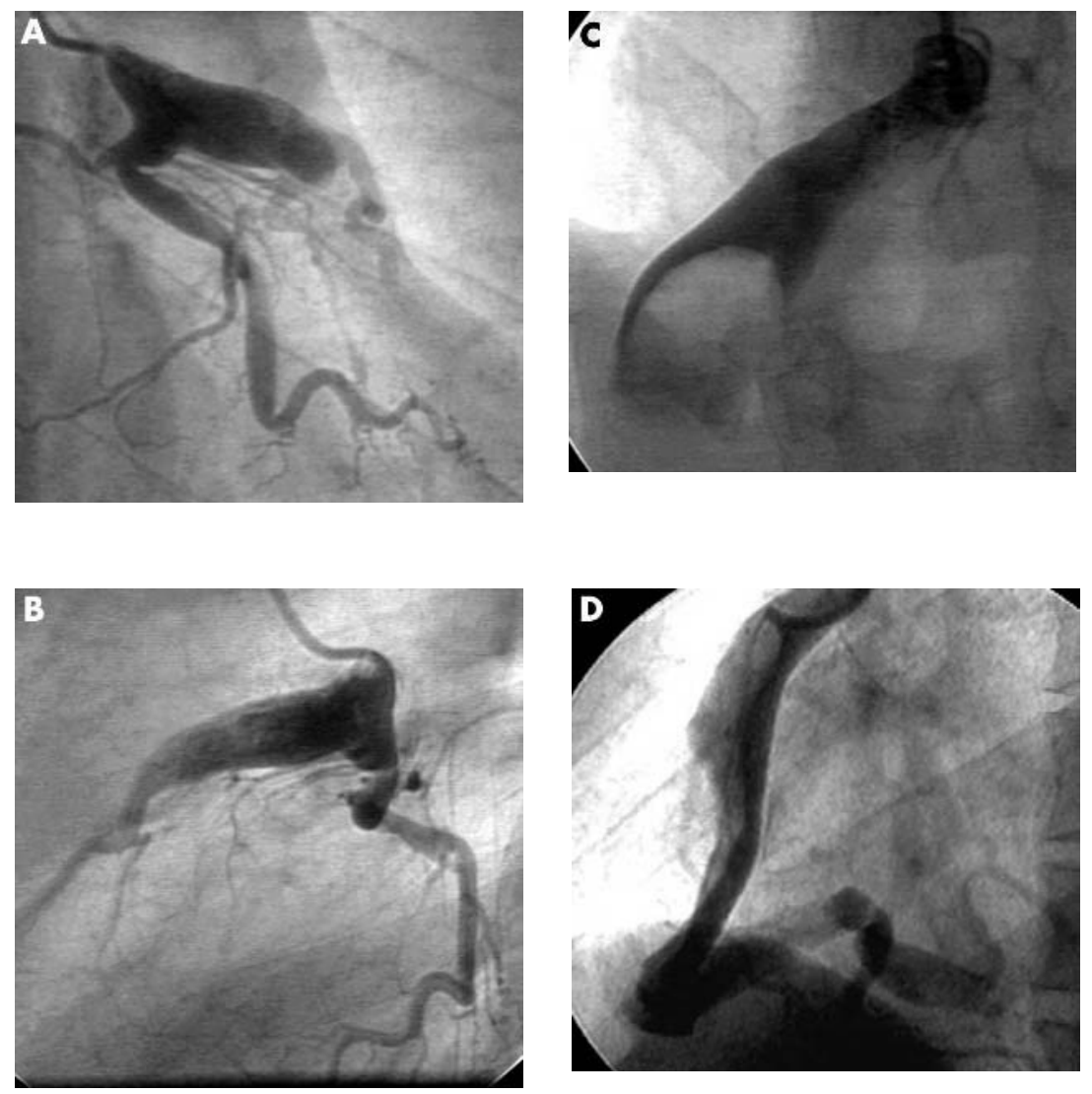

S S Deshpande $V$ Viswanathan NO Bansal drshantanud@yahoo.com 